University of Texas at El Paso

ScholarWorks@UTEP

\title{
Possible Explanation of Empirical Values of the Matern Smoothness Parameter for the Temporal Covariance of GPS Measurements
}

\author{
Gaël Kermarrec \\ Leibniz University Hannover, gael.kermarrec@web.de \\ Steffen Schön \\ Leibniz University Hannover, schoen@ife.uni-hannover.de \\ Vladik Kreinovich \\ The University of Texas at El Paso, vladik@utep.edu
}

Follow this and additional works at: https://scholarworks.utep.edu/cs_techrep

Part of the Mathematics Commons

Comments:

Technical Report: UTEP-CS-17-63

Published in Applied Mathematical Sciences, 2017, Vol. 11, No. 35, pp. 1733-1737.

\section{Recommended Citation}

Kermarrec, Gaël; Schön, Steffen; and Kreinovich, Vladik, "Possible Explanation of Empirical Values of the Matern Smoothness Parameter for the Temporal Covariance of GPS Measurements" (2017).

Departmental Technical Reports (CS). 1161.

https://scholarworks.utep.edu/cs_techrep/1161

This Article is brought to you for free and open access by the Computer Science at ScholarWorks@UTEP. It has been accepted for inclusion in Departmental Technical Reports (CS) by an authorized administrator of ScholarWorks@UTEP.For more information, please contact Iweber@utep.edu. 


\title{
Possible Explanation of Empirical Values of the Matérn Smoothness Parameter for the Temporal Covariance of GPS Measurements
}

\author{
Gaël Kermarrec ${ }^{1}$, Steffen Schön ${ }^{1}$, and Vladik Kreinovich ${ }^{2}$ \\ ${ }^{1}$ Geodetic Institute \\ Leibniz University of Hannover \\ Schneiderberg 50 \\ 30167 Hannover, Germany \\ gael.kermarrec@web.de, scheon@ife.uni-hannover.de \\ ${ }^{2}$ Department of Computer Science \\ University of Texas at El Paso \\ 500 W. University \\ El Paso, TX 79968, USA \\ vladik@utep.edu
}

\begin{abstract}
The measurement errors of GPS measurements are largely due to the atmosphere, and the unpredictable part of these errors are due to the unpredictable (random) atmospheric phenomena, i.e., to turbulence. Turbulence-generated measurement errors should correspond to the smoothness parameter $\nu=5 / 6$ in the Matérn covariance model. Because of this, we expected the empirical values of this smoothness parameter to be close to $5 / 6$. When we estimated $\nu$ based on measurement results, we indeed got values close to $5 / 6$, but interestingly, all our estimates were actually close to 1 (and slightly larger than 1 ). In this paper, we provide a possible explanation for this empirical phenomenon. This explanation is based on the fact that in the sensors, the quantity of interest is usually transformed into a current, and in electric circuits, current is a smooth function of time.
\end{abstract}

\section{Formulation of the Problem: An Empirical Fact That May Need Explaining}

Temporal covariance: general idea. In many practical situations, we perform repeated measurements of the corresponding quantity (or quantities) at different moments of time. 
Often, in data processing, measurement errors of different measurement results are assumed to be independent; see, e.g., [2]. In many cases, this assumption makes sense, since during the time between the two measurements the factors affecting the measurement change in a random way. However, when we make multiple repeated measurements, the time interval between the two consequent measurements is so small that at least some of these factors do not have time to change. As a result, there is a significant correlation between the measurement errors of two consequent measurements.

To properly process the results of the corresponding measurements, we need to know the covariance between measurement results obtained at different moments of time.

According to [3], in many practical applications, the covariance $C(T)$ between the measurements separated by time $T$ is often well described by the following Matérn model:

$$
C(T)=\varphi \cdot(\alpha \cdot T)^{\nu} \cdot K_{\nu}(\alpha \cdot T),
$$

for appropriate parameters:

- $\alpha$ (whose meaning is that it is the inverse of the correlation time) and

- $\nu$ (that describes the smoothness of the resulting process).

Here, $K_{\nu}(x)$ is the modified Bessel function of the second type of order $\nu$. In general, the Bessel function $J_{\alpha}(x)$ is defined as the solution to the differential equation

$$
x^{2} \cdot \frac{d^{2} y}{d x^{2}}+x \cdot \frac{d y}{d x}+\left(x^{2}-\alpha^{2}\right) \cdot y=0
$$

for which $y(0)=0$. Then, we define $I_{\alpha}(x) \stackrel{\text { def }}{=} \mathrm{i}^{-\alpha} \cdot J_{\alpha}(\mathrm{i} \cdot x)$, where $\mathrm{i} \stackrel{\text { def }}{=} \sqrt{-1}$, and

$$
K_{\alpha}(x) \stackrel{\text { def }}{=} \frac{\pi}{2} \cdot \frac{I_{-\alpha}(x)-I_{\alpha}(x)}{\sin (\alpha \cdot x)} .
$$

The Matérn covariance function can also be characterized by its Fourier transform - spectral density

$$
S(\omega)=\frac{\varphi \cdot 2^{\nu-1} \cdot \Gamma(\nu+1 / 2) \cdot \alpha^{2 \nu}}{\sqrt{\pi} \cdot\left(\alpha^{2}+\omega^{2}\right)^{\nu+1 / 2}},
$$

where $\Gamma(x)$ is the gamma-function.

Temporal correlation of GPS measurements: what we expected. For GPS measurements, the measurement error mostly comes from the uncertainty of atmospheric propagation, and this uncertainty, in its turns, is caused by turbulence. For turbulence, we expect the power spectrum to have asymptotics $S(\omega) \sim \omega^{-8 / 3}$ which corresponds to $\nu=5 / 6 \approx 0.83$; see, e.g., [1].

Of course, there are other factors affecting the measurement error, factors which are described by different smoothness values. Thus, we expected the 
empirical value of the smoothness parameter $\nu$ to be not necessarily equal to $\nu=5 / 6$, but close to this value.

Temporal correlation of GPS measurements: what we actually observed. In our analysis of the results of GPS measurements, we did indeed get values close to $5 / 6$ - in the sense that the value $5 / 6$ was always within the confidence interval for $\nu$. However, interestingly, all our Maximum Likelihood estimations of $\nu$ led to values between 1 and 1.05; see [1].

Again, as we have mentioned, this does not mean that there is any contradictions with the turbulence idea - the value $5 / 6$ is still within the confidence interval for $\nu$ - but the fact that in all the cases, we get values close to 1 and always larger than 1 may need explaining.

\section{A Possible Explanation}

Sensors usually transform a value of a quantity into an electric current. Most sensors - whether they are photo-sensors, temperature sensors, piezoelectric sensors - transform the quantity that we want to measure into an electric current. Then, based on the value of the current, we estimate the value of the desired physical quantity.

The current smoothly depends on time. In the linear approximation, any system that processes electric circuits can be viewed as consisting of resistors, capacitors, and inductors, the basic elements of all possible electric circuits. Here:

- the voltage of the resistor is proportional to the current $I$,

- the voltage of the capacitor is proportional to the electric charge - i.e., to the integral of the current, and

- the voltage of the inductor is proportional to the time derivative $\frac{d I}{d t}$ of the current.

Since voltage has to be always finite, this implies that the derivative of the current is always finite - i.e., that the dependence of the current on time is always differentiable.

This implies that the dependence of the measured value on time is also differentiable. Since the measured value of the quantity is determined based on the value of the corresponding current, this implies that the measured quantity should also be a differentiable function of time.

How is this related to the smoothness parameter $\nu$ ? It is known (see, e.g., [3], Section 2.4), that a random process described differentiable functions if and only if $\int \omega^{2} \cdot S(\omega) d \omega<+\infty$.

For the Matérn covariance function, we have $S(\omega) \sim \omega^{-(2 \nu+1)}$ for large $\nu$, so $\int \omega^{2} \cdot S(\omega) d \omega \sim \int \omega^{-(2 \nu-1)} d \omega$. For $\omega \rightarrow \infty$, this integral is infinite when $2 \nu-1 \leq 1$ : 
- when $2 \nu-1<1$, this integral is proportional to $\omega^{-(2 \nu-2)} \rightarrow \infty$, and

- when $2 \nu-1=1$, this integral is proportional to $\ln (\omega) \rightarrow \infty$.

The integral is finite when $2 \nu-1>1$, i.e., when $\nu>1$.

Thus, the fact that the dependence of the measured value on time is differentiable means that we should have $\nu>1$.

So what value $\nu$ should we expect? The actual value $\nu$ should be close to $5 / 6$. We want our measurements to be as accurate as possible, so we would like to have the value of the smoothness $\nu$ to be as close to $5 / 6$ as possible. On the other hand, as we have mentioned, we will always have values of the smoothness parameter larger than 1.

Out of the values larger than 1 , the smaller the value $\nu$-i.e., the closer it is to 1 - the closer it is to $5 / 6$.

So, for accurate measurements, we expect the corresponding value $\nu$ to be very close to 1 (but still larger than 1).

So, we have an explanation. This is exactly what we observe - we observe values $\nu$ which are close to 1 and larger than 1 . Thus, we indeed get a possible explanation for the observed phenomenon.

\section{Acknowledgments}

This work was performed when Vladik was a visiting researcher with the Geodetic Institute of the Leibniz University of Hannover, a visit supported by the German Science Foundation. This work was also supported in part by NSF grant HRD-1242122.

\section{References}

[1] G. Kermarrec and S. Schön, "A prior fully populated covariance matrices in least-squares adjustment - case study: GPS relative positioning", Journal of Geodesy, 2017, Vol. 91, No. 5, pp. 465-484.

[2] S. G. Rabinovich, Measurement Errors and Uncertainty: Theory and Practice, Springer Verlag, Berlin, 2005.

[3] M. L. Stein, Interpolation of Spatial Data: Some Theory for Krigging, Springer Verlag, New York, 1999. 